\title{
Les cinq sens, le corps et l'esprit
}

Chez les auteurs chrétiens de l'Antiquité et du Moyen Age, la tendance générale dans l'appréciation de la signification symbolique des cinq sens repose sur la considération de l'unité fondamentale, chez l'homme, entre le corps et l'esprit, permettant d'établir la doctrine des sens corporels et des sens spirituels. Le passage biblique que l'on est en droit de considérer comme le texte fondateur de cette conception de l'unité du corps et de l'esprit chez l'homme, en perspective chrétienne, est un extrait de la première épître aux Corinthiens : «Il y a diversité de dons de la grâce, mais c'est le même esprit ; diversité de ministères, mais c'est le même Seigneur ; diversité des modes d'action, mais c'est le même Dieu qui, en tous, met tout en oeuvre. À chacun est donnée la manifestation de l'Esprit en vue du bien de tous. À l'un, par l'Esprit, est donné un message de sagesse, à l'autre, un message de connaissance, selon le même Esprit ; à l'un, dans le même Esprit, c'est la foi ; à un autre, dans l'unique Esprit, ce sont les dons de guérison ; à tel autre, d'opérer des miracles, à tel autre, de prophétiser, à tel autre, de discerner les esprits, à tel autre encore, de parler en langues ; enfin à tel autre, de les interpréter. Mais tout cela, c'est l'unique et même Esprit qui le met en oeuvre, accordant à chacun des dons personnels divers, comme il veut. En effet, prenons une comparaison : le corps est un, et pourtant il a plusieurs membres : mais tous les membres du corps, malgré leur nombre, ne forment qu'un seul corps : il en est de même du Christ. Car nous avons tous été baptisés dans un seul Esprit en un seul corps, Juifs ou Grecs, esclaves ou hommes libres, et nous avons tous été abreuvés d'un seul Esprit. Le corps, en effet, ne se compose pas d'un seul membre, mais de plusieurs. Si le pied disait : « Comme je ne suis pas une main, je ne fais pas partie du corps ", cesserait-il pour autant d'appartenir au corps? Si l'oreille disait : «Comme je ne suis pas un oeil, je ne fais pas partie du corps », cesserait-elle pour autant d'appartenir au corps ? Si le corps entier était oeil, où serait l'ouïe ? Si tout était oreille, où serait l'odorat ? Mais Dieu a disposé dans le corps chacun des membres, selon sa volonté. Si l'ensemble était un seul membre, où serait le corps ? Il y a donc plusieurs membres mais un seul corps. L'oeil ne peut pas dire à la main : « Je n’ai pas besoin de toi », ni la tête dire aux pieds : «Je n’ai pas besoin de vous ». Bien plus,

Note: Les éléments traités dans cet article sont présentés de façon plus développée dans mon livre L'invention chrétienne des cinq sens dans la liturgie et l'art au Moyen Âge, Paris 2014.

Éric Palazzo, Université de Poitiers-CESCM, IUF

Ә Open Access. (C) 2020 Éric Palazzo, published by De Gruyter. (๕) BY-NC-ND This work is licensed under a Creative Commons Attribution-NonCommercial-NoDerivatives 4.0 International License.

https://doi.org/10.1515/9783110615937-004 
même les membres du corps qui paraissent les plus faibles sont nécessaires, et ceux que nous tenons pour les moins honorables, c'est à eux que nous faisons le plus d'honneur. Moins ils sont décents, plus décemment nous les traitons : ceux qui sont décents n’ont pas besoin de ces égards. Mais Dieu a composé le corps en donnant plus d'honneur à ce qui en manque, afin qu'il n'y ait pas de division dans le corps mais que les membres aient en un commun souci les uns des autres. Si un membre souffre, tous les membres partagent sa souffrance ; si un membre est glorifié, tous les membres partagent sa joie. Or vous êtes le corps $\mathrm{du}$ Christ et vous êtes ses membres, chacun pour sa part » (I Cor. XII, 4-27). En écho à ce passage, citons un autre extrait de la première épître aux Corinthiens où saint Paul associe le corps du chrétien à un temple de l'Esprit, également l'un des membres du Christ : "Ne savez-vous pas que vos corps sont les membres du Christ ? Prendrai-je les membres du Christ pour en faire des membres de prostituée ? Certes non ! Ne savez-vous pas que celui qui s'unit à la prostituée fait avec elle un seul corps ? Car il est dit : Les deux ne seront qu'une seule chair. Mais celui qui s'unit au Seigneur est avec lui un seul esprit. Fuyez la débauche. Tout autre péché commis par l'homme est extérieur à son corps. Mais le débauché pèche contre son propre corps. Ou bien ne savez-vous pas que votre corps est le temple du Saint-Esprit qui est en vous et qui vous vient de Dieu, et que vous ne vous appartenez pas ? Quelqu'un a payé le prix de votre rachat. Glorifiez donc Dieu par votre corps » (I Cor. VI, 12-20).

Dès les premiers siècles chrétiens, les théologiens et philosophes se font les relais de la conception grecque de l'homme où l'équilibre entre le corps et l'esprit, et même l'âme, est un souci majeur, suivant en cela les idées philosophiques de Platon d'un côté, et celles d'Aristote de l'autre ${ }^{1}$. Au III $^{\mathrm{e}}$ siècle, le néoplatonisme de Plotin (205-270) exerça une influence considérable sur la pensée chrétienne et notamment sur la manière dont le christianisme naissant pensait la relation entre l'âme et le corps chez l'homme ${ }^{2}$. Dans un célèbre passage extrait de ses Ennéades, Plotin établit une analogie entre le travail du sculpteur et celui produit par l'homme, en lui, dans son corps et dans son esprit pour se transformer en un être de beauté : "Reviens à toi-même et regarde : Si tu ne te vois pas toi-même beau, fais comme le sculpteur d'une statue qui doit devenir belle, il enlève, il gratte, il polit, il nettoie, jusqu'à ce qu'il fasse apparaître un beau visage dans la statue. Toi aussi, enlève tout ce qui est superflu, redresse ce qui est tortueux, nettoie ce qui est sombre, rends-le brillant, et ne cesse pas de sculpter

1 M.-Th. D’Alverny, «L'homme comme symbole. Le microcosme », Simboli e simbologia nell'alto medioevo, XXIII settimane di studio del centro italiano di studi sull'alto medioevo, t. I, Spolète 1975-1976, 123-195.

2 A. Grabar, Les origines de l'esthétique médiévale, Paris 1992. 
ta propre statue, jusqu'à ce que resplendisse pour toi la divine splendeur de la vertu, jusqu'à ce que tu voies « la sagesse debout sur son socle sacré » (citation du Phèdre de Platon). Es-tu devenu cela ? As-tu vu cela ? Est-ce que tu as toi-même un rapport pur, sans aucun obstacle à ton unification, sans que rien d'autre soit mélangé intérieurement avec toi-même ? Es-tu devenu tout entier une lumière véritable, non pas une lumière de dimension ou de formes mesurables qui peut diminuer ou augmenter indéfiniment de grandeur, mais une lumière absolument sans mesure, parce qu'elle est supérieure à toute mesure et à toute quantité ? Si tu te vois devenu cela, devenu toi-même une vision, prenant confiance en toimême, remontant déjà vers le haut, tout en restant ici-bas, n'ayant plus besoin de guide, fixe intensément les yeux et regarde ! ${ }^{3}$. Pour Plotin, l'homme a la capacité d'action sur sa beauté intérieure, comme s'il agissait, tel un sculpteur, afin d'atteindre l'harmonie et le corps et l'esprit et d'activer l'intensité de son sens visuel. À la même époque que le philosophe néo-platonicien, Lactance (250-325) est l'un des premiers à élaborer une conception de l'homme qui opère une synthèse entre la pensée antique et le christianisme ${ }^{4}$. Après avoir expliqué que l'anatomie de l'homme, notamment le squelette, était au service des vertus, dont la sagesse, Lactance disserte sur les cinq sens en considérant chacun des organes à l'origine des sensations. La tête de l'homme est comparée à une citadelle car elle est le siège de l'âme et les organes des sens, sauf le toucher, y résident. L'oeil est la fenêtre sur le monde qui permet à l'âme de voir l'environnement de l'homme. Les oreilles et le nez sont interprétés de la même façon que l'oeil. Au sujet de la bouche, Lactance souligne son importance pour l'émission de sons et pour le sens du goût qui sont mis au service de la connaissance comme de la louange de Dieu à travers les textes sacrés et par l'intermédiaire des lèvres qui chantent la gloire de Dieu. De cela, il ressort que, pour Lactance, la connaissance physiologique du corps de l'homme est centrale dans la définition de son anthropologie mais qu'elle doit être aussi appréhendée à partir de la signification symbolique des organes et des sens déjà teintée de théologie chrétienne. Une enquête approfondie et fort instructive menée sur le vocabulaire latin de la vie affective dans la littérature romaine des temps chrétiens à partir des mots et verbes relatifs à la perception sensorielle à permis d'aboutir à des conclusions identiques à celles de la conception chrétienne du corps par Lactance, opérant

3 Plotin, Ennéades, I, 6, 9, 7. Une interprétation de ce passage est proposé par J.-P. Deremble, «Penser l'articulation des contraires avec Plotin, une clé de l'église médiévale entre terre et ciel ", Matérialité et immatérialité dans l'Eglise au Moyen Âge. Actes du colloque de Bucarest, 22-23 octobre 2010, Bucarest 2012, 419-430, 423-424.

4 M. Perrin, L'homme antique et chrétien. L'anthropologie de Lactance, 250-325, Paris 1981, principalement 66 et suivantes. 
la symbiose entre la culture romaine antique et le christianisme naissant ${ }^{5} . \mathrm{Au} \mathrm{IV}$ siècle, saint Ambroise, évêque de Milan entre 374 et 397, a émis des idées similaires au sujet du corps de l'homme et de la valorisation de sa tête où se situe l'origine des perceptions sensorielles majeures telles que la vue et l'ouïe. Pour saint Ambroise toute l'activité sensorielle de l'homme part du cerveau auquel les organes des sens sont reliés par des nerfs. En d'autres termes, la tête est, si l'on ose l'expression, le coeur de l'émission et en même temps du ressenti des cinq sens $^{6}$. Certaines représentations de l'anatomie de l'homme dans des manuscrits de la seconde moitié du Moyen Âge montrent de fortes similitudes avec les idées de Lactance au sujet de la relation entre l'homme intérieur et l'homme extérieur ainsi que du point de vue de l'importance accordée à la tête, siège de l'âme et là où résident les principaux organes des sens ${ }^{7}$. Le III ${ }^{\mathrm{e}}$ siècle chrétien voit aussi naître le concept chrétien des cinq sens spirituels et leurs correspondances avec les cinq sens corporels ${ }^{8}$. Origène (vers 185-vers 253) a été l'initiateur du concept des sens spirituels qui porte principalement sur la réconciliation entre l'âme et le corps par l'établissement de correspondances entre les sens corporels et les sens spirituels dont le lien est établi par l'incarnation du Verbe : «Suivant les termes de l'Ecriture, il existe un genre de sens divin... Et ce genre comporte des espèces : la vue qui peut fixer des réalités supérieures au corps... ; l'ouïe percevant des sons dont la réalité n'est pas dans l'air ; le goût pour savourer le pain vivant descendu du ciel et donnant la vie au monde ; de même encore ces parfums dont parle Paul qui se dit " être pour Dieu la bonne odeur du Christ »; le toucher grâce auquel Jean affirme avoir touché de ses mains le logos de vie. Ayant trouvé le sens divin, les bienheureux, les prophètes regardaient divinement, écoutaient divinement,

5 P. Morillon, Sentire, sensus, sententia. Recherche sur le vocabulaire de la vie intellectuelle, affective et physiologique en latin, Lille 1974.

6 Saint Ambroise, Hexameron, Lib. VI, PL 14, 281 : « Caput itaque oculis explorat omnis, auribus occulta rimatur, cognoscit abscondita, audit quid aliis agatur in terris...Quanto igitur major humani capitis ornatus est, qui cerebrum nostrum hoc est, sedem originemque nostrorum sensuum capillis capitis munit et vestit... »; 283 : «Itaque propter oculos ferunt, medendi periti cerebrum hominis in capite locatum, alios autem nostri corporis sensus propter cerebrum finitimo quodam esse domicilio constitutos...unde omnes nervi, et quo referunt universa, que vel oculos viderit, vel auris audierit, vel odor inhalaverit, vel lingua increpuerit, vel os sopris acceperit ». 7 Sur le dessin d'un manuscrit conservé à Cambridge (University Library, Gg 1.1), réalisé au XIV siècle, M. Camille, « Before the Gaze. The Internal Senses and Late Medieval Practices of Seeing », dans R. S. Nelson (éd.), Visuality before and beyond the Renaissance. Seeing as others saw, Cambridge 2000, 197-221 et, plus récemment, M. Carruthers, «Intention, sensation et mémoire dans l'esthétique médiévale », Cahiers de civilisation médiévale 55 (2012), 367-378, 372-374.

8 Sur les sens spirituels et leur évolution dans la pensée chrétienne de l'Antiquité et du Moyen Âge : P. L. Gavrilyuk et S. Coakley (éds.), The Spiritual Senses. Perceiving God in Western Antiquity, Cambridge 2012. 
goûtaient et sentaient de la même façon, pour ainsi dire d'un sens qui n'est pas sensible ${ }^{9}$. La puissance de la pensée d'Origène au sujet des sens spirituels aura été nécessaire pour défendre les cinq sens corporels dont la raison d'être repose sur leurs liens, en l'homme, avec les sens intérieurs. Il faut dire que les sens corporels ont été, très tôt, violemment mis en cause par de grands auteurs chrétiens, tel saint Jérôme (vers 347-420). Dans son traité contre Jovinien, il stigmatise les cinq sens qui sont des fenêtres permettant aux vices de pénétrer dans l'âme. Et saint Jérôme d'ajouter à son rejet des cinq sens, véritables portes d'entrée du mal en l'homme voués à perturber son âme, une condamnation sans appel du sens tactile qui perd le genre humain dans la volupté. Nombre de théologiens au Moyen Age ont, par la suite, oeuvré avec succès pour la revalorisation du sens du toucher ${ }^{10}$. Le concept des sens spirituels tente de valoriser les sens corporels en soulignant qu'ils n'ont de raison d'être que dans leur relation avec l'homme intérieur animé par ses sens spirituels. De façon générale, le constat est celui de la volonté de plusieurs auteurs chrétiens de l'Antiquité pour engager un processus de valorisation de l'homme intérieur qui se reflète dans l'homme extérieur.

Comme pour la plupart des domaines de la théologie, la pensée de saint Augustin a exercé une influence considérable sur la conception chrétienne des cinq sens impliquant une réflexion approfondie sur la relation entre le corps et l'esprit dans le christianisme. En premier lieu, on lui doit l'approfondissement du concept des sens spirituels lui ayant permis de forger le thème du sens intérieur ou du sens du coeur. En second lieu, saint Augustin a donner d'importants développements à la hiérarchie au sein des cinq sens. Il est également celui qui a construit le thème essentiel de la synesthésie et de ses modes de fonctionnement. Pour résumer, on peut affirmer que la pensée augustinienne relative aux cinq sens représente le jalon majeur dans l'histoire du thème et, surtout, dans le processus de leur christianisation ${ }^{11}$. La théorie augustinienne des cinq sens est

9 Origène, Contre Celse, I, 48, cité par M. Canévet, « Sens spirituel », Dictionnaire de spiritualité, t. XIV, Paris 1990, col. 600.

10 N. Largier, «Tactus spiritualis. Remarques sur le toucher, la volupté et les sens spirituels au Moyen Âge », Micrologus XIII, 2005, 233-249. Pour les citations de saint Jérôme extraites de son Adversus Jovinianum, 233 : «Per quinque sensus, quasi per quasdam fenestras, vitiorum ad animam introitus est...Horum perturbationibus anima praegravatur : et capitur aspectu, auditu, odoratu, sapore, tactu...Tactus autem alienorum corporum, et feminarum ardentior appetitus, vicinus insaniae est. Igitur cum per has portas, quasi quidam perturbationum cunei ad arcem nostrae mentis intraverint, ubi erit libertas, ubi fortitudo ejus, ubi de Deo cogitatio : maxime cum tactus depingat sibi etiam praeteritas voluptates, et recordatione vitiorum cogat animam compati, et quodammodo exercere quod non agit ?...».

11 Pour une vue d'ensemble de la pensée augustinienne sur les cinq sens : D. Chisterer, «Symbolism and the Senses in saint Augustine », Religion 14 (1984), 31-51; E. Vance, "Seeing God : 
dominée par le thème de l'incarnation du Verbe et de son parallèle avec l'activation, chez l'homme, du sens du coeur et de son extériorisation grâce aux sens corporels $^{12}$. Dans l'un des ses multiples sermons, saint Augustin s'exprime en ces termes au sujet de l'incarnation du Verbe et de l'activation du sens du coeur chez l'homme : «J'essaie de parler du Verbe ; le verbe, la parole de l'homme pourrait peut-être nous offrir quelque chose de semblable. Cependant quelle distance, quelle disproportion ! Il n'y a pas de parité ; et cependant une certaine ressemblance me permettra d'insinuer quelque lumière à vos esprits. Voici la parole que je vous adresse, je l'ai eue d'abord dans mon cœur ; elle est parvenue jusqu'à vous, mais elle ne m'a point quitté, ce qui n'était pas en vous a commencé d'y être ; cette parole s'est dirigée vers vous, mais elle est restée au-dedans de moi. De même donc ma parole est venue frapper vos sens sans quitter mon coeur, ainsi le Verbe s'est manifesté à nous sans quitter le sein de son Père. Ma parole était en moi, elle en est sortie pour devenir voix ; le verbe de Dieu était dans le sein du Père, il en est sorti pour se faire chair. Mais puis-je faire de ma voix ce qu'il a pu faire de sa chair ? Je ne puis retenir ma voix qui s'envole ; pour lui, non seulement il a retenu, conservé sa chair dans sa naissance, dans sa vie, dans toutes ses actions, mais il l'a ressuscitée après sa mort, et il a fait monter jusqu'au Père ce char sur lequel il était venu jusqu'à nous. Appelez la chair de Jésus-Christ un vêtement ; appelez-là un char ; appelez-là une monture, comme il n'a pas dédaigné de l'indiquer lui-même dans la parabole du Samaritain... " ${ }^{13}$. Pour saint Augustin, les sensibilia peuvent être assimilés à des signes au sens théologique du terme, c'est-à-dire à des effets visibles de la manifestation du divin sur terre et chez l'homme dont le point culminant est l'incarnation du Verbe. Tout ceci peut s'exercer à condition néanmoins de considérer que le sens du coeur est le seul vrai juge de la perception du monde extérieur par l'homme. Dans sa conception de la synesthésie et de la définition des cinq sens telles qu'ils apparaissent exposés dans le livre X des Confessions, saint Augustin insiste également sur la hiérarchie permettant de classer les cinq sens selon un ordre favorisant la vue et l'ouïe. Ces deux sens tiennent un rôle de premier plan pour la conversion et la révélation de Dieu à l'homme dans les textes bibliques. Au sujet de l'oeil et de la vue, premier dans la hiérarchie des cinq sens, saint Augustin affirme l'existence

Augustine, Sensation and the Mind's Eye », dans S. G. Nichols, A. Kablitz et A. Calhoun (éds.), Rethinking the Medieval Senses : heritage, fascinations, frames, Baltimore 2008, 13-29 et, dans une perspective plus axée sur la dimension sonore, B. Holsinger, Music, Body and Desire in Medieval Culture, Stanford 2001, 61-83.

12 G. Bavaud, « Un thème augustinien : le mystère de l'incarnation, à la lumière de la distintion entre le verbe intérieur et le verbe proféré », Revue des études augustiniennes 9 (1963), 95-101. 13 Saint Augustin, Sermon 119, PL 38, 675-676, cité et traduit dans l'article de Bavaud, ibid., 97. 
des yeux du coeur qui permettent aux saints de voir Dieu dans l'au-delà grâce à l'incarnation qui a donné la possibilité aux hommes de voir le Christ en corps : «Gardons-nous donc de prétendre qu'en l'autre vie les saints ne pourront voir Dieu, les yeux fermés, Dieu, qu'ils verront toujours de l'Esprit. Mais le verront-ils aussi des yeux du corps, lorsqu'ils les auront ouverts, c'est la question. Car, si leurs yeux tels qu'ils seront, spirituels en un corps spirituel, n'ont pas une autre vertu que ces yeux tels que nous les avons aujourd'hui, assurément ils seraient impuissants à voir Dieu ; leur puissance sera donc infiniment différente, si par eux l'on voit cette nature incorporelle qui n'est point contenue en un lieu, tout entière partout. Quoique nous disions en effet que Dieu est au ciel et sur la terre ; dirons-nous qu'une partie de lui-même est au ciel, et une autre sur la terre ? Il est tout entier dans le ciel, tout entier sur la terre, non pas alternativement, mais tout à la fois, chose impossible à toute nature corporelle. Cette vue sera donc infiniment plus puissante ; et ce n'est pas à dire qu'elle l'emporte en pénétration sur celle que l'on attribue à certaines espèces d'aigles ou de reptiles (car quelle que soit la clairvoyance des animaux, ils ne peuvent voir que des corps), mais ce sera l'excellence de ces yeux, de voir même l'incorporel. Et peut-être est-ce cette vue pénétrante qui en ce corps mortel aura pour un moment été donnée aux yeux du saint homme Job, quand il dit à Dieu : «Mon oreille d'abord avait entendu ; et maintenant mon oeil te voit ; et c'est pourquoi je me suis méprisé moi-même et la honte m'a consumé, et je me suis vu n'être que terre et que cendre ». Bien que ceci puisse sans difficulté s'entendre de l'oeil de l'esprit, ces yeux dont l'apôtre dit : «Qu'il éclaire les yeux de votre coeur ». Or, que Dieu ne se voie de ces yeuxlà, c'est ce dont ne doute aucun chrétien qui reçoit d'un coeur fidèle cette parole de notre divin maître : «Bienheureux les coeurs purs car ils verront Dieu ». Mais Dieu se verra-t-il aussi des yeux du corps, c'est la question que nous agitons ${ }^{14}$. A plusieurs reprises dans son petit traité intitulé « De videndo Deo », saint Augustin exprime des idées proches de celles développées dans ce dernier passage de La Cité de Dieu ${ }^{15}$. Dans son préambule, il commence par soutenir que l'invisibilité de Dieu se voit par les yeux du corps et que c'est aussi toute l'intériorité de l'homme qui perçoit le Verbe de Dieu. Pour les autres sens également, saint Augustin explique, toujours dans ce petit traité consacré aux différentes façons de voir Dieu, que les sens corporels ne font qu'exprimer la sensation intérieure de l'homme, de nature spirituelle. Le processus de correspondance entre l'homme intérieur et les sens corporels décrit par saint Augustin ne peut se réaliser sans

14 Saint Augustin, La Cité de Dieu, Livre XXII, chap. XXIX, t. III, 349-350.

15 Saint Augustin, De videndo Deo, PL 33, 596-618. 
la force de la volonté, véritable moteur, chez l'homme, de sa mise en place et de sa réalisation ${ }^{16}$.

$\mathrm{Au}$ final, le parcours proposé par saint Augustin dans l'exploration par l'homme de ses sens corporels et de son intériorité, c'est-à-dire de l'esprit de l'homme, là où se trouve le sens du coeur, débouche sur la parfaite correspondance entre l'élément extérieur et le mystère intérieur. Tel est le sens qu'il faut accorder à la célèbre formule de saint Augustin tiré du chapitre 80 de son Traité sur l'évangile de Jean : "La parole va à la rencontre de l'élément et le sacrement s'opère ${ }^{17}$. Par certains côtés, la formule augustienne fait écho à celle contenue à la fin du chapitre 19 de la règle de saint Benoît. Le chapitre 19 de la règle conclut cet ensemble sur l'office. En voici le contenu : « Nous avons la certitude que Dieu est partout présent et que les yeux du Seigneur regardent les bons et méchants en tout lieu. Nous devons, sans aucun doute, en être bien plus certains encore, quand nous prenons part au service de Dieu. Aussi, rappelons-nous toujours ce que dit le Prophète : «Servez le Seigneur avec crainte », et encore, " Psalmodiez avec sagesse »; et : " Je psalmodierai pour toi sous le regard des anges ». Réfléchissons donc à l'attitude qui s'impose sous le regard de Dieu et de ses anges, et, en psalmodiant, soyons tels que notre esprit soit en accord avec notre voix ${ }^{18}$. Dans ce chapitre, la phrase de conclusion - « ut mens nostra concordet voci nostrae »-devenue un adage repris dans certains textes officiels de l'Église $\mathrm{au} \mathrm{XX}{ }^{\mathrm{e}}$ siècle, suggère la concordance parfaite entre l'esprit et la voix au moment

16 Ibid., 599-600 : «Si quis vero mihi indicet voluntatem suam, cuius os et vox mihi praesens est ; tamen quia ipsa voluntas quam mihi indicat, latet sensum corporis et animi mei, credo, non video : aut si eum mentiri existimo, non credo, etsi forte, ut dicit, ita sit ...Voluntatem vero eius a quo audit ut credat, et ipsam Christi resurrectionem non videt, sed credit ».

17 Saint Augustin, Tract. In Iohan. 80, 2-3 (« Accedit verbum ad elementum et fit sacramentum »), I. Renaud-Chamska, « De la sensibilité aux choses et du sens des mots. La bénédiction de l'eau baptismale », La Maison-Dieu 188 (1991), 41-55, 42.

18 La règle de saint Benoît, texte latin-français, tr. et éd. H. Rochais, Paris 1980, 62-63 : « Ubique credimus divinam esse praesentiam et oculos Domini in omni loco speculari bonos et malos, maxime tamen hoc sine aliqua dubitatione credamus, cum ad opus divinum adsistimus. Ideo semper memores simus quo ait Propheta : Servite Domino in timore, et iterum : Psallite sapienter, et : In conspectu angelorum psallam tibi. Ergo consideremus qualiter oporteat in conspectu Divinitatis et angelorum et eius esse, et sic stemus as psallendum, ut mens nostra concordet voci nostrae ». En écho à ce passage de la règle bénédictine, citons un extrait du pontifical romain du XIII siècle pour l'ordination du chantre ou du psalmiste : « Le psalmiste, autrement dit le chantre, après avoir été instruit par l'archidiacre, peut recevoir son office sans que l'évêque en soit informé, sur le seul commandement du prêtre, qui lui dit : «Veille à croire en ton coeur ce que tu chantes de ta bouche, et ce que tu crois en ton coeur, confirme-le par tes actes ", Le pontifical de la curie romaine au XIII ${ }^{e}$ siècle, texte latin, traduction et introduction par M. Goullet, G. Lobrichon et É. Palazzo, Paris 2004, 37. 
de la pratique de la psalmodie par le moine. Elle souligne aussi que l'esprit doit être en accord avec la voix qui est première dans l'exécution de la psalmodie. À travers ce précepte, saint Benoît recommande aux moines de chanter les psaumes et à laisser l'esprit s'en imprégner. Autrement dit, pour saint Benoît, la priorité est accordée à la voix, au son, ou, plus largement, à l'oral et au corporel, qui doit ensuite activer l'esprit, l'intériorité de l'Homme. Ce précepte de la règle de saint Benoît considère comme essentielle l'idée selon laquelle, dans la liturgie monastique, les sens corporels - ici, il s'agit du son de la voix - jouent un rôle déterminant dans l'activation de la perception du divin par l'esprit. La compréhension intérieure et intelligible du divin passe pour l'Homme par l'activation des sens corporels dans le cadre de la liturgie. La façon dont saint Benoît considère l'harmonie entre le corps et l'esprit dans la pratique de la psalmodie monastique fait écho à ce qu'expriment plusieurs grandes figures de la théologie chrétienne de l'Antiquité. À lire la phrase de saint Augustin tirée de son traité sur l'évangile de Jean, on ne peut s'empêcher d'y déceler la même signification que celle exprimée par la phrase finale du chapitre 19 de la règle de saint Benoît. Il en est de même dans ce passage du sermon 227 de l'évêque d'Hippone : «Que se réalise dans la conscience ce que montrent les lèvres " (sicut ostendunt labia, fiat in conscientia $)^{19}$. Dans ces textes, c'est bien l'élément corporel, le sens physique qui sert de guide à l'esprit dans l'accès au divin et pour la perception de l'Invisible. Les termes employés par saint Jean Chrysostome, au IV siècle, dans un passage de son "Incompréhensibilité de Dieu » résument idéalement cette position défendue par les théologiens de l'Antiquité au sujet de l'activation de l'esprit de l'homme à partir de celle de ses sens corporels afin d'atteindre l'accord parfait entre le corps et l'esprit : « Je peux, disent-ils, prier aussi dans ma maison, tandis qu'il m'est impossible d'entendre chez moi une homélie ou un sermon. Tu te trompes toi-même, homme! Si tu peux en effet prier à la maison, tu ne saurais y prier de la même façon qu'à l'église, où se trouve un si grand nombre de pères spirituels et où une clameur unanime monte vers Dieu. Quand tu invoques le Seigneur dans ton particulier, tu n'es pas exaucé aussi bien que lorsque tu le fais en compagnie de tes frères. Car, il y a ici quelque chose de plus, à savoir l'accord des esprits et des voix. Le lien de la charité et les prières des prêtres. Car les prêtres président afin que les prières de la foule, qui sont plus faibles, recevant le renfort des leurs, qui sont plus fortes, s'élèvent avec elles vers le ciel ${ }^{20}$.

19 Saint Augustin, Sermo 227, PL 38, 1109.

20 F. Cassingena-Trévédy, Les Pères de l'Eglise et la liturgie, Paris 2009, 50-51. 
Un dernier aspect de la pensée de saint Augustin sur les cinq sens mérite d'être mentionné. Dans l'usage qu'il fait du vocabulaire sensitif, il n'est pas rare de voir l'évêque d'Hippone employer un verbe « sensoriel » pour exprimer une idée spirituelle. Tel est par exemple le cas concernant le verbe "tangere » (toucher) qu'Augustin emploie pour désigner l'acte contemplatif consistant à « toucher » Dieu ${ }^{21}$. On rencontre ici l'usage métaphorique des cinq sens corporels dont l'activation est au service de celle d'actes spirituels reflétant le parcours intérieur de l'homme vers Dieu. En même temps, Amalaire de Metz, au IX ${ }^{\mathrm{e}}$ siècle, ouvre la voie en direction de l'appréciation fondamentalement sensorielle de l'action liturgique et de sa finalité spirituelle. Dans son commentaire sur le verset de l'alleluia, l'exégète carolingien suggère que, dans l'exécution des actes de la liturgie, la mise en action des sens opère aussi bien au niveau du corps que de celui de l'homme intérieur et de ses sens spirituels. Au contraire, pour bon nombre d'auteurs de l'époque carolingienne et jusqu'au XI ${ }^{\mathrm{e}}$ siècle, la tendance dominante est celle de la valorisation des cinq sens à partir d'un regard, plutôt favorable, porté sur le corps humain en général. Dans quelques-uns de ses écrits, Jean Scot Erigène, au IX ${ }^{\mathrm{e}}$ siècle, soutient fermement la valorisation des cinq sens du corps de l'homme étant donné leur capacité à être les relais actifs de la connaissance intime de Dieu chez l'homme. Par exemple, dans son commentaire sur le prologue de l'évangile de saint Jean, Scot Erigène exprime en ces termes sa considération favorable envers les cinq sens corporels par l'activation conjointe de l'intériorité et de l'extériorité de l'homme, du corps et de l'esprit : « La voix de l'Aigle mystique retentit aux oreilles de l'Eglise. Puissent nos sens, du dehors, en recueillir le son fugitif, et notre esprit, au-dedans de nous, en pénétrer la signification durable ! Voix de l'oiseau de haut vol, non de celui qui planerait au-dessus de l'air matériel ou de l'éther, voire au-dessus de tout l'univers sensible, mais de celui qui, par les ailes rapides d'une profonde théologie - les regards d'une lumineuse et haute contemplation -, s'élève au-dessus de toute théorie, au delà de toutes les choses qui sont et de toutes celles qui ne sont pas ${ }^{22}$. Plus loin dans le même commentaire sur le prologue de l'évangile de Jean, Scot Erigène précise un peu plus sa pensée au sujet des cinq sens et ce qu'ils permettent pour la connaissance de Dieu par l'homme : " La lumière de la divine connaissance s'est retirée du monde lorsque l'homme s'est écarté de Dieu. Et c'est d'une double manière que la lumière éternelle se fait connaître elle-même au monde : par l'Ecriture et par les créatures. Car la divine connaissance ne peut être restaurée en nous que par les

21 P. Adnès, « Toucher, touches », Dictionnaire de spiritualité, Paris 1974, 1073-1098, 1075.

22 J. S. Erigène, Homélie sur le prologue de Jean, introduction, texte et traduction de E. Jeauneau, Paris 1969, 201-205. 
lettres de l'Ecriture et par le spectacle des créatures. Etudie les paroles de l'Ecriture et, dans ton esprit, comprends-en la signification : tu y découvriras le Verbe. Par tes sens corporels, observe les formes et la beauté des choses sensibles : en elles, ton intelligence reconnaîtra le Verbe de Dieu...Et, bien que ce deuxième monde soit situé au niveau le plus bas de l'univers, le Verbe était cependant en lui, et c'est le Verbe qui l'a fait. Il est aussi le premier échelon que doivent gravir ceux qui veulent s'élever par les sens à la connaissance de la vérité, car le spectacle des choses visibles entraîne l'esprit qui raisonne vers la connaissance des choses invisibles ${ }^{23}$.

Dans l'ensemble, la seconde moitié du Moyen Âge connut un regain d'intérêt pour la métaphore ancienne de l'homme-citadelle dont les sens sont comparés aux portes et aux fenêtres lui donnant accès au monde extérieur et à sa connaissance. Au XI ${ }^{\mathrm{e}}$ siècle, dans l'une de ses lettres, Pierre Damien pousse assez loin la métaphore entre le corps de l'homme et ses cinq sens et la citadelle aux cinq portes. Pour lui, l'existence de l'homme est une citadelle assiégée qui doit se défendre contre les tentations extérieures du monde. Et l'homme-citadelle a cinq portes puisque le corps est encerclé par les cinq sens. Pour Pierre Damien, les meilleures armes défensives contre les mauvaises influences du monde sensible sont des citations bibliques qu'il énumère après avoir rappelé le besoin, pour l'homme, de mettre des serrures et des verrous aux portes que sont les cinq sens : «Mettons-y des serrures, mettons-y des verrous, des barres et des loquets, pour protéger jalousement l'accès à nos sens de l'irruption des vices et des vanités mondaines ${ }^{24}$. Pour Pierre Damien et d'autres auteurs à la même époque, les sens corporels ne doivent pas faire entrer en l'homme les vices extérieurs mais permettre le développement des vertus, de nature spirituelle. Telle est la signification qu'il faut comprendre d'un passage d'un texte de Bruno de Segni au XI siècle qui met en correspondance les vertus avec, si ce n'est une vertu, du moins une action positive chez le chrétien. Par exemple, pour cet auteur, c'est dans le toucher que réside l'âme de la foi car le sens tactile permet de connaître Dieu. De son côté, la vue entraîne l'homme vers l'intelligence des choses tandis que l'odorat offre la possibilité aux chrétiens de distinguer la doctrine catholique de

23 Erigène, Homélie, 259 et 293.

24 Pierre Damien, PL 144, 325-326 : « Nam velut in quinque portarum civitate consistimus, dum in corpore, quod quinque sensibus cingitur, habitamus. His ergo portis seras apponimus, his repagula, vectes ac pessulos adhibemus, cum sensuum nostrorum aditus a vitiis irruventibus ac mundi vanitatibus sollicite custodimus » et, un peu plus loin, « Has itaque corporalium sensuum portas, dilectissimi, et vitiorum irrventium phalanges obstruite, et virtutum spiritualium agminibus aperite » $(P L$ 144, 330) ; cf. J.-Y. Tilliette, « Le symbolisme des cinq sens dans la littérature morale et spirituelle des XI ${ }^{\mathrm{e}}$ et XII ${ }^{\mathrm{e}}$ siècles », Micrologus X (2002), 15-32, 23. 
l'hérésie par la bonne et la mauvaise odeur ${ }^{25}$. Des mises en garde contre les effets néfastes pour l'homme des cinq sens sont également pointés chez Odon d'Asti au $\mathrm{XI}^{\mathrm{e}}$ siècle dans son commentaire sur le psautier. Pour lui, les cinq sens sont des portes qui doivent refléter les vertus et permettre au diable de ne pas pénétrer en l'homme ${ }^{26}$. Pour Sicard de Crémone, les fenêtres de l'église sont comparées aux cinq sens qui sont les portes de la vie ou bien celles de la mort ${ }^{27}$. Dans la sphère de l'harmonie musicale, au XI ${ }^{\mathrm{e}}$ siècle, le théoricien de la musique, Gui d'Arezzo, exprime de façon particulièrement saisissante les idées destinées à valoriser les cinq sens corporels en relation avec le modèle de l'architecture : « Il n'est pas étonnant que la variété des sons charme l'oreille, puisque la variété des couleurs est agréable l'œil, la variété des odeurs délecte l’odorat et la langue se réjouit du changement des saveurs. Ainsi, en effet, par les fenêtres du corps, la douceur des choses qui s'y adaptent pénètre merveilleusement dans les demeures secrètes du cœur ${ }^{28}$. S'engageant dans la même voie que le théoricien de la musique du $\mathrm{XI}^{\mathrm{e}}$ siècle, Guillaume Durand, le principal exégète de la liturgie au XIII ${ }^{\mathrm{e}}$ siècle est préoccupé par le coeur de l'homme où s'opèrent le dialogue entre les mouvements de la chair et l'action de l'Esprit-Saint, ainsi qu'il l'exprime dans la première explication qu'il donne de l'autel : « L'autel signifie la mortification de nos sens ou notre coeur, dans lequel les mouvements de la chair sont consultés par l'ardeur de l'Esprit-Saint ${ }^{29}$. Pour saint Bernard (1090-1153) le thème central de sa compréhension de la dimension sensorielle tourne autour de l'idée que l'union entre Dieu et les hommes se réalise fondamentalement par l'intermédiaire des

25 Tilliette, ibid., 25. Le texte de Bruno de Segni se trouve dans PL 165, 280-281. On peut rapprocher le sens moral accordé par Bruno de Segni aux cinq sens de celui que Raban Maur, au IX ${ }^{\mathrm{e}}$ siècle, avait soutenu pour les mouvements du corps (De universo, Lib. VI, cap. II, PL 118, 178-179). Dans ce texte, l'abbé de Fulda, s'appuyant lui aussi sur des citations bibliques, expose la signification positive des gestes et mouvements du corps destinés à exprimer les vertus telles que la foi et l'humilité.

26 Odon d'Asti, PL 135, 1198 : « Attollite portas, principes vestras ». Unusquisque homo quinque portas habet ; habet enim visum, auditum, gustum, odoratum et tactum. Hae autem portae, nisi singulis virtutibus, quasi singulis custodibus, custodiantur, per eas diabolus frequenter ingreditur et regreditur : sed, quoniam praedicta generatio iam Dominum quaerit, et diabolus et eius portas ab hominibus fugerunt, portaeque aeternales levatae sunt, quia soli aeterno Regi praedicti sensus aperiuntur ».

27 Sicard de Crémone, Mitralis de officiis, éd. G. Sarbak et L. Weinrich, Turnhout 2008, 15 : « Vel per fenestras, quae clausae turbinem excludunt, patulae includunt, intellige quinque sensus corporis, qui circumcisi sunt ianua vite, laxivi sunt ostia mortis ».

28 Gui d’Arezzo, Micrologus, trad. M.-N. Colette et J.-C. Jolivet, Paris 1996, 60-61.

29 Guillaume Durand, Rationale divinorum officiorum, éd. A. Davril et T. Thibodeau, Turnhout 1995, 29 : « Rursus altare est mortificatio nostra seu cor nostrum in quo carnales motus fevore spiritus sancti consumuntur ». 
cinq sens. Saint Bernard affirme l'expérience individuelle de Dieu chez chacun de nous car, dit-il, nous aimons les choses spirituelles à travers « le charnel ». Dans ce sens, il met en avant l'apport essentiel de l'incarnation qui, d'une certaine manière, autorise l'homme à aimer les choses charnelles en vue de la connaissance de Dieu et de développer son amour pour lui. Pour saint Bernard donc, la discussion sur les cinq sens est une affaire d'amour car ce sont bien les sens corporels qui nous aident à aimer Dieu et ses créations ${ }^{30}$. La conception de saint Bernard sur les cinq sens, plutôt favorable à leur intégration dans la catégorie des éléments permettant à l'homme de connaître et d'aimer Dieu, est tempérée par des attaques virulentes contre l'effet sensoriel provoqué par la beauté de l'art. Tel est le sens de la critique de l'abbé de Clairvaux envers les sens dans le cadre plus général de sa célèbre Apologie.

Un célèbre dessin contenu dans un manuscrit réalisé à l'abbaye allemande d' Heilbronn au XII ${ }^{\mathrm{e}}$ siècle résume à lui seul une large partie des éléments explorés sur la relation entre le corps et l'esprit dans la théologie chrétienne du Moyen Âge à partir de l'exploration des cinq sens. Comme on va le voir, il suggère également une réflexion approfondie sur la dimension sensorielle dans le parcours de l'homme sur terre et dans la perspective de celui qu'il aura à accomplir dans l'audelà, guidé par le modèle du Christ et les vertus chrétiennes (Fig. 1) ${ }^{31}$. Le manuscrit est conservé à la bibliothèque universitaire d'Erlangen (Allemagne) (ms. 8) et a sans doute été réalisé à l'abbaye d'Heilbronn au XII ${ }^{\mathrm{e}}$ siècle, en Franconie. Il est composé de 157 feuillets de parchemin et contient différents textes de l'Ancien Testament parmi lesquels l'Ecclésiaste et le Cantique des cantiques ou bien encore, une version glosée du livre des lamentations attribué à Jérémie. La célébrité de ce manuscrit tient principalement en la présence de cinq dessins en pleine page représentant des thèmes iconographiques en relation avec la figure $\mathrm{du}$ roi Salomon, comme ceux des folios $3 \mathrm{v}$ et $68 \mathrm{v}$ montrant respectivement Salomon, une première fois, entouré de huit personnages qu'il est en train d'enseigner et, une seconde fois, ouvrant le livre de l'Ecclésiaste, Salomon en tant

30 Sur toutes ces questions, G. Rudy, Mystical language of Sensation in the Later Middle Ages, New York-Londres 2002, 45-65. R. Fassetta, « Le corps dans l'anthropologie monastique de saint Bernard », Cîteaux. Commentarii cistercienses 63 (2012), 91-107.

31 Les études sur ce dessin ne sont pas légion. Parmi elles, mentionnons E. Lutze, Die Bilderhandschriften der Universitätsbibliothek Erlangen, Erlangen 1936, 3-4 ; C. Nordenfalk, « Les cinq sens dans l'art du Moyen Âge », Revue de l'art 34 (1976), 17-28, en partic. 18-19; C. Heck, L'échelle céleste. Une histoire de la quête du ciel, Paris 1999, 87-89 ; J. Jung, « The Tactile and the Visionary : Notes on the Place of Sculpture in the Medieval Religious Imagination », dans C. Hourihane (éd.), Looking Beyond. Visions, Dreams and Insights in Medieval Art and History, Princeton 2010, 203-240, 208-209 et A. Trivellone, L'hérétique imaginé. Hétérodoxie et iconographie dans l'Occident médiéval, de l'époque carolingienne à l'inquisition, Turnhout 2009, 298-309. 


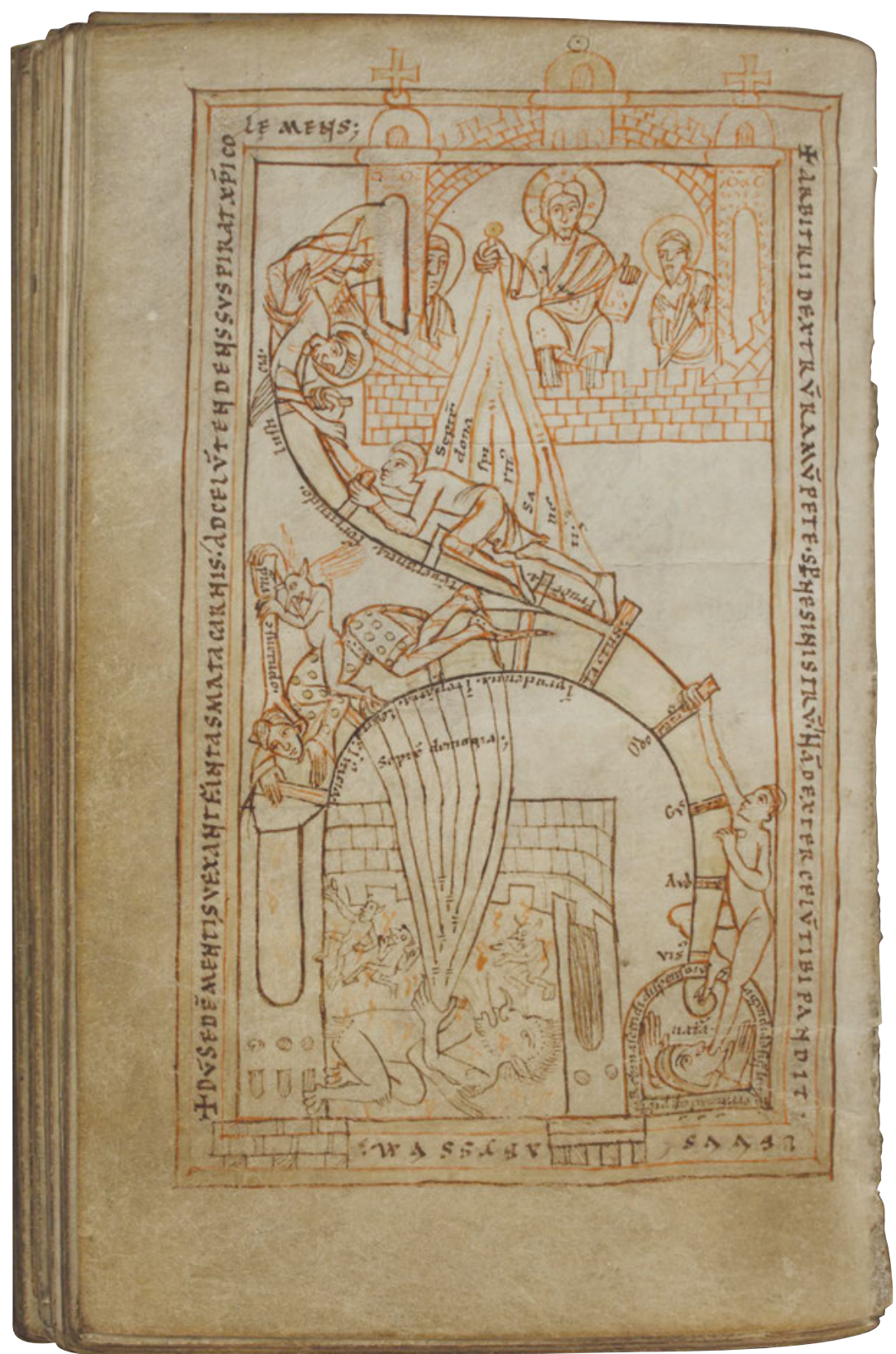

Fig. 1: Erlangen, Universitätsbibliothek, ms. 8, fol. 130v (@ Erlangen, Universitätsbibliothek). 
que roi entouré par huit personnages. Le dessins du folio $129 \mathrm{v}$ montre un thème en relation avec le livre des lamentations puisqu'on y voit le prophète Jérémie ayant la vision de la destruction et de l'incendie de Jérusalem. Au folio 130r, l'iconographie du dessin représente le thème de l'Eglise luttant contre les agressions verbales et physiques du monde. Faisant suite à la vision de Jérémie de la destruction de Jérusalem, le dessin du folio 130r représente le Christ dans une mandorle, entouré par saint Pierre et saint Paul, supporté, au registre inférieur de la composition, par la personnification de l'Eglise attaquée par ses ennemis - dont un hérétique - à coups de lance et d'épée ainsi que de paroles agressives transcrites dans les phylactères. Sur le folio 130v, on voit un dernier dessin en pleine page où le rôle des cinq sens dans le parcours de l'homme sur terre et dans l'au-delà est essentiel. À certains égards, l'iconographie du dessins du folio 130v poursuit la réflexion sur le thème de la persécution de l'Eglise, ou, de façon plus générale, du combat entre le bien et le mal, qui est au centre de l'image du folio 130v. On y a représenté le thème du chemin de vie des bons et des méchants ou des deux voies de la vie humaine. En bas à droite de la composition, le buste de la personnification de la nature fait sortir de sa bouche un homme nu qui entame l'escalade d'une échelle dont les cinq premiers barreaux sont assimilés aux cinq sens par des inscriptions. Dans la partie médiane de l'image, l'échelle se sépare en deux voies offertes à l'homme pour continuer son chemin sur terre et dans l'au-delà. À la croisée des chemins, l'homme peut choisir entre le bien et le mal. Le personnage qui a choisi la voie du mal est chevauché par un diablotin qui le pousse vers le bas de l'image à l'aide d'une fourche sur laquelle est inscrite, en latin, la maxime « comportement dépravé ». Il n'échappera à l'observateur attentif que le personnage qui a fait le choix du « comportement dépravé » est vêtu comme un prince et qu'il continue "l'escalade » de son échelle en s'appuyant sur des barreaux identifiés à l'imprudence, l'intempérance, l'inconstance et l'injustice. Dans la partie inférieure de l'image, le « mauvais homme » est attendu en enfer par des diablotins et le diable lui-même qui tient dans la main gauche des sortes de phylactères faisant allusion aux «sept démons » qui sont le pendant négatif des sept dons de l'Esprit-Saint représentés, aussi sous la forme de phylactères, dans les mains du Christ trônant en majesté dans le registre supérieur de la composition. Les inscriptions logées dans le cadre de l'image soulignent le sens positif du côté droit et le sens négatif du côté gauche. Revenons au parcours de «l'homme bon » qui a fait le choix de la foi en Dieu et au Christ. Pour cet homme, simplement vêtu, le chemin se poursuit à la croisée de l'échelle prenant naissance dans la bouche de la Nature, en direction du Christ, trônant au centre d'une architecture symbolique - on pense alors à la Jérusalem céleste défendue par le Seigneur, en opposition à la ville attaquée du folio 130r - et entouré de deux personnages, peut-être la Vierge Marie et saint Pierre. Pour lui permettre l'accès à la cité céleste, 
l'homme qui a emprunté la voie chrétienne est aidée par les barreaux de l'échelle assimilées aux vertus cardinales : la prudence, la tempérance, la force et la justice. Au sommet de l'échelle, un ange l'attend pour l'aider à accomplir son voyage et à accéder à la vision du Christ trônant en majesté au centre de la ville céleste. Le Christ lui-même intervient pour faciliter le voyage du « bon chrétien » puisqu'il lui transmet les sept dons de l'Esprit-Saint matérialisés par les phylactères qu'il tient dans sa main droite. On dirait même que le Christ tire vers lui l'homme en train d'escalader « son » échelle céleste pour l'aider physiquement et pas seulement spirituellement à le faire parvenir jusqu'au paradis. L'iconographie du folio 130v du manuscrit d'Heilbronn est, à plusieurs égards, un cas unique dans les images chrétiennes du Moyen Âge. Elle s'inspire vraisemblablement de plusieurs thèmes iconographiques dont ceux de l'échelle céleste de Jacob et d'Hercule à la croisée des chemins, d'origine antique. À ces thèmes iconographiques revisités pour la circonstance, l’image associe le thème plus général du combat des vices et des vertus évoqué à travers les cinq sens. On peut également voir dans la représentation du thème des deux voies possibles pour l'homme l'inspiration du passage de l'évangile de saint Matthieu : «Entrez par la porte étroite. Car large est la porte et spacieux est le chemin qui mènent à la perdition, et il en est beaucoup qui entrent par là ; mais étroite est la porte et resserré le chemin qui mènent à la vie, et il en est peu qui les trouvent " (Matth VII, 13-14). Comme l'a fait remarquer Christian Heck, le sens global de l'iconographie du dessin est proche de la description du monde faite par Hildegarde de Bingen au XII ${ }^{\mathrm{e}}$ siècle où l'accent est porté sur le choix de la bonne voie pour l'homme à partir des deux chemins qui s'offrent à lui : «Que l'homme résiste à la volupté de la chair, de peur qu'il ne se laisse entraîner par les délices de ce monde...car il est pèlerin et son père l'attend...C'est pourquoi, ô homme, si tu tournes ton regard vers les deux voies, c'est-à-dire vers le bien et le mal, alors tu t'instruis...Ecoutez donc, ô hommes, et ne perdez pas de vue l'entrée de la Jérusalem céleste ... Maintenant donc regarde ces deux sentiers, l'un vers l'Orient, l'autre vers l'aquilon...si tu as recours à Satan...alors tu tomberas dans la perdition $»^{32}$. Dans le choix auquel l'homme est confronté sur son parcours de la vie humaine, les cinq sens peuvent jouer un rôle de guides bienveillants ou bien, au contraire, perdre l'homme et le mener droit en enfer. Saint Bernard a comparé les cinq sens à un échelon permettant de s'élever jusqu'à l'invisible « Grand est celui qui, usant des sens comme d'un échelon, arrive à s'élever, par la philosophie, jusqu'à l'invisible $»^{33}$. La proximité entre cette idée et l'iconographie du dessin du manuscrit

32 Hildegarde de Bingen, Scivias, I, 4 et II, 3, cité par Heck, L'échelle céleste, 88-89. 33 Saint Bernard, De consideratione, V, 3, cité par Heck, L'échelle céleste, 87. 
allemand permet d'envisager l'influence de la pensée de saint Bernard sur la conception de l'image. De façon générale, on observe de fortes correspondances entre les grandes tendances de la théologie chrétienne des cinq sens et l'iconographie du dessin. En premier lieu, les cinq sens font partie intégrante de l'homme et peuvent être utilisés par lui dans un sens positif ou négatif. L'image du manuscrit allemand reflète l'idée courante chez les théologiens chrétiens selon laquelle les cinq sens peuvent, selon ce que l'homme décide d'en faire, servir le bien ou le mal. Selon les cas, les cinq sens contribuent au chemin menant vers Dieu par la connaissance des choses invisibles à travers le monde sensible ou bien, perdent l'homme sur le chemin du vice. Pour faire ce choix, l'homme est face à lui-même. Comme le suggère saint Augustin et d'autres auteurs chrétiens avec lui, la décision de l'homme d'emprunter la voie positive qui le mènera vers Dieu repose sur son intention et sa volonté qui, comme on l'a vu précédemment, peuvent agir positivement sur les sens corporels. D'une certaine manière, le dessin du manuscrit allemand montre en second lieu cela : l'homme, composé de ses cinq sens corporels, a la choix de faire son ascension vers le Seigneur grâce à sa volonté et à son intention et d'activer ainsi les cinq sens de façon positive pour connaître Dieu. À partir de là, il est aidé par le Christ lui-même et les dons de l'Esprit-Saint. Il n'est pas sans intérêt de constater que, le concepteur de l'image a établi un parallèle entre les cinq premiers échelons de « l'échelle-chemin ", assimilés aux cinq sens, et les quatre derniers, identifiés aux quatre vertus cardinales. Autrement dit, la voie qui mène l'homme vers Dieu et qu'il a la choix d'emprunter, s'il le décide, grâce à l'intention et à la volonté, est jalonnée d'échelons positifs qui l'aideront dans son parcours : les cinq sens et les vertus cardinales. Mais les cinq sens formant les échelons du début du chemin peuvent aussi être négatifs et être complétés par les échelons négatifs de la voie qui mène vers l'enfer. L'ordre hiérarchique dans lequel les échelons des cinq sens apparaissent semble refléter une partie de la pensée chrétienne à ce sujet. En effet, la vue et l'ouïe ouvrent la voie pour guider l'homme vers le bon chemin. Ensuite, le relais est pris par l'odorat et le goût. Enfin, le dernier échelon est celui du toucher dont on a vu qu'il était, pour plusieurs auteurs, le moyen privilégié pour l'homme de connaître Dieu et de réaliser son ascension vers lui. Dans l'iconographie de notre image, on est frappé par le détail montrant le personnage empruntant la voie chrétienne avec le détail de son pied droit touchant le dernier échelon des cinq sens - le toucher comme s’il était en train de s'élancer vers la voie de Dieu en prenant appui sur le sens tactile. Au contraire de cela, l'homme qui a opté pour l'autre voie - celle qui le mènera en enfer - n'a plus de contact physique avec le dernier échelon des cinq sens, le toucher. Enfin, l'iconographie du dessin allemand fait du thème de l'ascension à la fois corporelle et spirituelle de l'homme grâce aux cinq sens le sujet central de l'image, ajoutant à cela l'idée que les cinq sens, utilisés à bon escient 
par l'homme grâce à sa volonté et à son intention, participent pleinement à l'expérience du divin et à la connaissance de Dieu, au même titre que les vertus cardinales. Pour lui permettre d'accéder à la vision du Christ trônant en majesté au sein de la cité céleste, l'homme gravit les échelons d'une échelle, mettant en quelque sorte l'accent sur les degrés qu'il doit franchir pour arriver au terme de son chemin et obtenir la récompense qui lui est dûe. Le thème de l'ascension spirituelle de l'homme, par degrés, est pour une grande part fondée sur l'épisode de l'échelle de Jacob, dans le livre de la Genèse, dont on a vu qu'il avait pu inspirer l'iconographie du dessin du manuscrit allemand. Pour certains commentateurs de la liturgie au Moyen Âge, dont Guillaume Durand au XIII ${ }^{\mathrm{e}}$ siècle, la signification théologique des degrés - dans le texte du Rational de Guillaume Durand, il s'agit des marches qui mènent à l'autel - renvoie non seulement au thème de l'échelle de Jacob mais aussi à celui des vertus - les marches - permettant de monter à l'autel : « Le prophète nous montre continuellement, dans les quinze psaumes, les degrés que l'homme saint a élevés dans son coeur. Jacob vit cette échelle dont le haut touchait les cieux. Par ces degrés, sont entendus, d'une manière convenable et claire, les degrés des vertus par lesquels on monte à l'autel, c'est-à-dire au Christ, selon cette parole du psalmiste : « Et ils marcheront et s'élèveront de vertu en vertu ». Et Job : «J'annoncerai le Seigneur dans quelque rang que Dieu me place, et à quelque degré qu'il me fasse asseoir " ${ }^{34}$. L'idée centrale développée par Guillaume Durand dans son commentaire exégétique au sujet des marches qui mènent à l'autel, c'est-à-dire le Christ, présente de fortes similitudes avec celle qui semble avoir largement inspirée le dessin réalisé à l'abbaye d'Heilbronn au XII ${ }^{\mathrm{e}}$ siècle. C'est par degrés que l'homme fait son ascension spirituelle vers le Christ en s'inspirant de l'échelle de Jacob et guidé par la voix des prophètes et du psalmiste. Dans le commentaire de Guillaume Durand, les degrés sont assimilés aux vertus comme le montre le dessin puisque les quatre derniers échelons de l'échelle menant l'homme vers Dieu sont les vertus cardinales. Certes, le commentateur de la liturgie du XIII ${ }^{\mathrm{e}}$ siècle n'associent pas les

34 Guillaume Durand, Rationale divinorum officiorum, 33-34 : «Ceterum gradus quibus altare ascenditur spiritualiter demonstrant apostolos et martyres Christi quos, quia pro eius amore sanguinem suum fuderunt, sponsa in canticis amors vocat ascensum purpureum. Exprimunt quoque quindecim virtutes qui significantur per quindecim gradus quibus ascendebatur in templum Salomonis, et a propheta in quindecim psalmis continue demonstrantur quos beatus vir ascensiones in corde suo disposuit. Hanc scalam vidit Iacob, sumitas eius celos tangebat. Per hos ergo gradus competenter virtutum gradus intelliguntur quibus ad altare, id est Christum, ascenditur, iuxta illud psalmi : « Et ambulabunt de virtute in virtutem ». Et Iob : « Per singulos gradus meos pronuntiabo illum ». Des idées similaires sont exprimées par Sicard de Cremone, Mitralis de officiis, 12. 
cinq sens aux degrés sur lesquels il disserte. Malgré cela, on est en droit de supposer que, dans l'image du manuscrit allemand, les cinq sens sont aussi considérés comme des vertus, au même titre que les autres échelons - les quatre vertus cardinales - de l'échelle " positive » empruntée par l'homme à condition qu'il manifeste le désir d'accéder à la vision de Dieu par une bonne intention et grâce à sa volonté. 
